\title{
Metamodel-based multi-objective robust design optimization of structures
}

\author{
J. Martínez-Frutos \& P. Martí-Montrull \\ Structural Optimization Group, Technical University of Cartagena, Spain
}

\begin{abstract}
Multi-objective robust optimization (MORO) is a highly demanding computational task because of the direct nesting of the uncertainty quantification within optimization. This work presents an approach based on Kriging models to efficiently include the uncertainty quantification in the optimization procedures. In the proposed approach the metamodels appear both at optimization level as well as at uncertainty quantification level. The proposed methodology allows us to: (1) assess the robustness of each design using a reduced number of simulator runs, as compared with conventional approach procedures; and (2) to decouple the uncertainty quantification of the optimization, allowing us to solve the problem with a lower computational cost compared to the nested approach. A benchmark problem has been considered using different approaches in order to compare their relative merits. The results show that the proposed method has potential to obtain solutions with reasonable accuracy and a considerably lower number of function calls than required by conventional methods.

Keywords: efficient multi-objective robust optimization, evolutionary algorithms, Kriging models.
\end{abstract}

\section{Introduction}

In structural engineering, the designer often has to deal with problems that involve conflicting objectives and sources of uncertainty in diverse structural parameters, such as geometric imperfections, material properties or applied loads. The set of optimal solutions obtained using conventional deterministic methods can be very sensitive to perturbations in design variables, leading to a deterioration of the optimal structural performance or even to a set of infeasible designs. Hence, it is desirable to obtain a set of optimal solutions which are less sensitive to variations 
in the structural parameters. The Multi-Objective Robust Optimization (MORO) takes into account the robustness of each design in the optimization process, leading to a set of robust solutions known as Pareto robust solutions [1].

Among the different approaches that address the MORO problem, authors such as Deb and Gupta [2] incorporate the robustness of the objective function within the optimization problem without considering the uncertainty in the constraints. Conversely, Gunawan and Azarm [1] and Li et al. [3] take into account both the robustness of the objective functions as well as the robustness of the constraints. Gunawan and Azarm [1] and Li et al. [3] propose a double loop optimization approach with interval uncertainty. These approaches can be computationally intractable, especially in high-dimensional problems [4], since two optimization problems need to be resolved for each design. In order to reduce the computational cost, some authors such as Hu et al. [4] and Shimoyama et al. [5] suggest the use of approximations to assist the multi-objective optimization problem. In this respect, Hu et al. [4] propose a two-level optimization with uncertainty intervals assisted by Kriging models. In the work proposed by Shimoyama et al. [5], Kriging models are used to obtain mean and standard deviation of the objective functions efficiently. The methodology proposed by Shimoyama et al. [5] doubles the number of objective functions compared to the deterministic approach. Said methodology can be inefficient in high-dimensional problems. The former approaches provide a set of robust solutions, but do not identify what the degree of robustness against other design is [6]. Recently, Erfani and Utyuzhnikov [6] presented a methodology suitable for obtaining solutions with different levels of robustness by introducing an additional objective function.

This work presents an approach based on Kriging models to efficiently include the uncertainty quantification in the optimization procedures. The proposed methodology allows: (1) to appraise the robustness of each design using a reduced number of simulator runs compared with conventional approach procedures; (2) to solve the MORO problem in a single-loop optimization assisted by Kriging models; and (3) to obtain multiple solutions to the MORO problem with different levels of robustness of both the objective functions and the constraints, without additional simulator calls.

\section{Metamodel-based multi-objective robust optimization}

\subsection{Multi-objective robust optimization}

The conventional multi-objective optimization problem can be formulated as follows:

$$
\begin{aligned}
& \min _{\mathbf{x} \in \mathbb{R}^{\mathbf{n}}}\left\{f_{1}(\mathbf{x}, \mathbf{p}), \ldots, f_{m}(\mathbf{x}, \mathbf{p})\right\} \\
& \text { s.t. } g_{j}(\mathbf{x}, \mathbf{p}) \leq 0 j=1, \cdots, m_{i} \\
& \text { s.t. } h_{k}(\mathbf{x}, \mathbf{p})=0 k=1, \cdots, m_{k} \\
& \quad \mathbf{x}^{\text {lower }} \leq \mathbf{x} \leq \mathbf{x}^{\text {upper }}
\end{aligned}
$$


where $\mathbf{x} \in \mathbb{R}^{\mathrm{n}}$ is the vector of design variables to be optimized; $\mathbf{p} \in \mathbb{R}^{\mathrm{q}}$ is the vector of non-design parameters; $\mathrm{x}^{\text {lower }}$ and $\mathrm{x}^{\text {upper }}$ are the lower and the upper bounds of the design variables respectively; $g_{j}$ and $h_{k}$ are the problem inequality and equality constraints. The solution of the multi-objective problem defined in (1), leads to a set of optimal solutions in the Pareto sense: A vector $\mathbf{x}$ is called a Pareto solution to problem (1) if another design vector $\mathbf{x}^{*}$ does not exist in the feasible space such that, $f_{i}\left(\mathbf{x}^{*}, \mathbf{p}\right) \leq f_{i}(\mathbf{x}, \mathbf{p})$ for any $i=1, \cdots, m$, and $j \in\{1, \cdots, m\}$ : $f_{j}\left(\mathbf{x}^{*}, \mathbf{p}\right)<f_{j}(\mathbf{x}, \mathbf{p})$ exists.

The conventional multi-objective optimization problem considers all the variables and parameters involved in the problem as being deterministic. For that reason, the optimal performance of the structure can be dramatically degraded in the presence of sources of uncertainty. The Multi-Objective Robust Optimization (MORO) takes into account the robustness of each design in the optimization process, leading to a set of robust solutions known as Pareto robust solutions [1].

Among the different approaches that address the MORO problem, in this paper the formulation proposed by Mattson and Messac [7] is considered. The mean values of the design metrics plus the standard deviations are minimized as follows:

$$
\begin{aligned}
& \min _{\mathbf{x}, \mathbf{p}, \in \mathbb{R}^{\mathrm{n}}}\left\{\mu_{f_{1}(\mathbf{x}, \mathbf{p}, \boldsymbol{\xi})}+k \sigma_{f_{1}(\mathbf{x}, \mathbf{p}, \boldsymbol{\xi})}, \ldots, \mu_{f_{m}(\mathbf{x}, \mathbf{p}, \boldsymbol{\xi})}+k \sigma_{f_{m}(\mathbf{x}, \mathbf{p}, \boldsymbol{\xi})}\right\} \\
& \text { s.t. } \\
& \mu_{g_{j}(\mathbf{x}, \mathbf{p}, \boldsymbol{\xi})}+k \sigma_{g_{j}(\mathbf{x}, \mathbf{p}, \boldsymbol{\xi})} \leq 0 \quad j=1, \cdots, m_{i} \\
& \quad \mathbf{x}^{\text {lower }} \leq \mathbf{x} \leq \mathbf{x}^{\text {upper }}
\end{aligned}
$$

where $\xi$ is a random variable vector that represents the uncertainty of the problem (design variables and/or parameters); $\mu_{f_{i}}$ and $\sigma_{f_{i}}$ are the mean and the standard deviation of the objective function $f_{i}$; and $\mu_{g_{j}}$ and $\sigma_{g_{j}}$ are the mean and the standard deviation of the inequality constraints respectively. All the equality constraints are transformed into inequality constraints to obtain multiple solutions to the MORO problem with different levels of robustness of both the objective functions and constraints.

In order to solve the problem formulated in 2 , it is necessary to combine optimization techniques with uncertainty quantification techniques. The most straightforward approach is to perform an uncertainty quantification loop for each iteration of the optimization algorithm. However, this can lead to an intractable problem. For this reason, diverse approaches exist in the literature that aim to break the nested relationship between uncertainty quantification and optimization [8]. In this work we investigate the used of Kriging models to efficiently include the uncertainty quantification in the MORO procedures.

\subsection{Kriging models}

A metamodel replaces a computationally expensive simulation model called a simulator by an emulator which is much faster to evaluate. Among the different metamodeling techniques, the Kriging models [9] have gained increasing popularity in recent years for use in engineering design, owing to the flexibility 
in capturing nonlinear behaviours [10], and the ability to provide statistical information on the predicted output [11].

Kriging models assume that the simulator can be approximated by a sample path of a Gaussian stochastic process $\mathscr{G}(\mathbf{x})$ whose prior mean $E[\mathscr{G}(\mathbf{x})]=\mathbf{f}(\mathbf{x})^{\mathrm{T}} \boldsymbol{\beta}$ and prior covariance function $\operatorname{Cov}\left[\mathscr{G}(\mathbf{x}), \mathscr{G}\left(\mathbf{x}^{\prime}\right)\right]=\alpha^{2} \mathrm{R}\left(\mathbf{x}, \mathbf{x}^{\prime}, \boldsymbol{\phi}\right)$ are unknown. In the former expressions; $\boldsymbol{\beta}=\left[\beta_{1}, \ldots, \beta_{\mathrm{p}}\right]^{\mathrm{T}}$ is a vector of unknown parameters; $\boldsymbol{f}(\mathbf{x})=\left[f_{1}(\mathbf{x}), \ldots, f_{\mathrm{p}}(\mathbf{x})\right]^{\mathrm{T}}$ is a set of known functions of $\mathbf{x} \in \mathbb{R}^{\mathrm{n}} ; \alpha^{2}$ is the variance of $\mathscr{G}(\mathbf{x})$; and $R\left(\mathbf{x}, \mathbf{x}^{\prime}, \boldsymbol{\phi}\right)$ is the autocorrelation function between $\mathbf{x}$ and $\mathbf{x}^{\prime}$. The stochastic process $\mathscr{G}(\mathbf{x})$ represents the prior knowledge of the simulator, for that reason the choice of the autocorrelation model should be made consistently with the known properties of the simulator. The most widely used class of autocorrelation function is the anisotropic generalized exponential model:

$$
\mathrm{R}\left(\mathbf{x}, \mathbf{x}^{\prime}, \boldsymbol{\phi}\right)=\exp \left(\sum_{\mathbf{i}=\mathbf{1}}^{\mathbf{n}}-\frac{\left|\mathbf{x}_{\mathbf{i}}-\mathbf{x}_{\mathbf{i}}^{\prime}\right|^{s}}{\phi_{\mathbf{i}}}\right), \quad \mathbf{1} \leq s \leq 2
$$

The parameters $\boldsymbol{\beta}, \alpha^{2}$ and $\phi$ are unknown a priori and are determined from the set of simulator responses $\mathscr{Y}=\left\{y_{1}, \ldots, y_{m}\right\}$ which are evaluated onto an experimental design $\mathscr{X}=\left\{\mathbf{x}_{1}, \ldots, \mathbf{x}_{m}\right\}$. Using Bayesian techniques, the posterior distribution of $\mathscr{G}(\mathbf{x})$ conditional on the vector of observations $\mathscr{Y}=$ $\left\{y_{1}, \ldots, y_{m}\right\}$ is also Gaussian [12] with mean value:

$$
\hat{y}(\mathbf{x}) \equiv \mathrm{E}[\mathscr{G}(\mathbf{x}) \mid \mathscr{Y}]=\mathbf{f}(\mathbf{x})^{\mathrm{T}} \hat{\boldsymbol{\beta}}+\mathbf{r}(\mathbf{x})^{\mathrm{T}} \mathbf{R}^{-1}\left(\mathscr{Y}^{\mathrm{T}}-\mathbf{F} \hat{\boldsymbol{\beta}}\right)
$$

and variance:

$$
\begin{aligned}
\operatorname{Cov}\left[\mathscr{G}(\mathbf{x}), \mathscr{G}\left(\mathbf{x}^{\prime}\right) \mid \mathscr{Y}\right] & =\alpha^{2}\left\{R\left(\mathbf{x}, \mathbf{x}^{\prime}, \boldsymbol{\phi}\right)-\mathbf{r}(\mathbf{x})^{\mathrm{T}} \mathbf{R}^{-1} \mathbf{r}\left(\mathbf{x}^{\prime}\right)+\right. \\
& \left.+\mathbf{u}(\mathbf{x})^{\mathrm{T}}\left(\mathbf{F}^{\mathrm{T}} \mathbf{R}^{-1} \mathbf{F}\right)^{-1} \mathbf{u}\left(\mathbf{x}^{\prime}\right)\right\}
\end{aligned}
$$

where we have introduced:

$$
\begin{aligned}
\mathbf{u}(\mathbf{x}) & =\boldsymbol{f}(\mathbf{x})-\mathbf{F}^{\mathrm{T}} \mathbf{R}^{-1} \mathbf{r}(\mathbf{x}) \\
\hat{\boldsymbol{\beta}} & =\left(\mathbf{F}^{\mathrm{T}} \mathbf{R}^{-1} \mathbf{F}\right)^{-1} \mathbf{F}^{\mathrm{T}} \mathbf{R}^{-1} \mathscr{Y} \\
\alpha^{2} & =\frac{1}{m}(\mathscr{Y}-\mathbf{F} \hat{\boldsymbol{\beta}})^{\mathrm{T}} \mathbf{R}^{-1}(\mathscr{Y}-\mathbf{F} \hat{\boldsymbol{\beta}}) \\
F_{\mathrm{ij}} & =f_{j}\left(\mathbf{x}_{\mathrm{i}}\right), \quad i=1, \ldots, m, j=1, \ldots, p \\
R_{\mathrm{ij}} & =\mathrm{R}\left(\mathbf{x}_{\mathrm{i}}, \mathbf{x}_{\mathrm{j}}, \boldsymbol{\phi}\right), \quad i=1, \ldots, m, j=1, \ldots, m \\
r_{\mathrm{ij}}(\mathbf{x}) & =\mathrm{R}\left(\mathbf{x}_{\mathbf{i}}, \mathbf{x}_{\mathrm{j}}, \boldsymbol{\phi}\right), \quad i=1, \ldots, m, j=1, \ldots, m
\end{aligned}
$$

$\hat{y}(\mathbf{x})$ represents the best prediction of the simulation model for a point $\mathbf{x}$, and $\operatorname{Cov}[\mathscr{G}(\mathbf{x}), \mathscr{G}(\mathbf{x}) \mid \mathscr{Y}]$ is the predicted error variance which provides probabilistic confidence intervals in addition to the prediction.

In (4) and (5) $\hat{\boldsymbol{\beta}}$ and $\alpha^{2}$ are derived analytically and only depend on the autocovariance parameters $\phi$. In the present work, the correlation parameters are 
determined using maximum likelihood estimation (MLE) [12], solving the global optimization problem:

$$
\max \left(-\frac{m \ln \left(\alpha^{2}\right)+\ln (|\mathbf{R}|)}{2}\right)
$$

In this work, a set of different instances of Kriging are created using different correlation and regression functions. The best model of the set is selected using the mean square errors, in particular the root mean square error (RMSE) and the $R^{2}$. Both the $R M S E$ and the $R^{2}$ are estimated using a global cross-validation errors measure called prediction sum of squares (PRESS) [13] without additional simulator calls:

$$
\operatorname{PRESS}_{\mathrm{RMSE}}=\sqrt{\frac{\sum_{i=1}^{m}\left(\hat{y}_{i}-\hat{y}_{-i}\right)^{2}}{m}}, \operatorname{Pred} R^{2}=1-\frac{\sum_{\mathrm{i}=1}^{m}\left(\hat{\mathrm{y}}_{\mathrm{i}}-\hat{\mathrm{y}}_{-\mathrm{i}}\right)^{2}}{\sum_{\mathrm{i}=1}^{m}\left(\hat{\mathrm{y}}_{\mathrm{i}}-\overline{\mathrm{y}}\right)^{2}}
$$

$\hat{y}_{i}$ is the prediction at the design point $i, \bar{y}$ is the mean of the predicted values and $\hat{y}_{-i}$ is calculated by removing each point $i$ from the modeling points, constructing a new Kriging model at the remaining points and obtaining the prediction at the removed point.

\subsection{Proposed approach}

This work presents an approach based on Kriging models that approximate the statistical moments on the design domain and efficiently include the uncertainty quantification in the optimization loop. The scheme of the proposed approach is depicted in figure 1.

The proposed method consists in a two-stage framework. The first stage aims at constructing a set of Kriging models that approximate the statistical moments of both the objective functions and the constraints on the design domain $(\boldsymbol{\mu}(\mathbf{x}), \boldsymbol{\sigma}(\mathbf{x})$, $\gamma(\mathbf{x}), \boldsymbol{\beta}(\mathbf{x}))$. The second stage consists in the resolution of the multi-objective problem using deterministic algorithms. In this work an evolutionary algorithm (NSGA-II) was used to fulfilled this target. The two-stage framework is further detailed below:

Stage 1: setting up Kriging models

1. Create a design of experiment (DoE) in the design variable domain $\mathbf{X}=$ $\left\{\mathbf{x}_{1}, \cdots, \mathbf{x}_{i}, \cdots, \mathbf{x}_{m_{x}}\right\}$. Latin Hypercube Sampling (LHS) was used for this.

(a) For each design point $\mathbf{x}_{i}$ a new design of experiments is generated in the random variables domain $\left\{\boldsymbol{\xi}_{1}, \cdots, \boldsymbol{\xi}_{j}, \cdots, \boldsymbol{\xi}_{m_{\xi}}\right\}$.

(b) Each point $\left\{\mathbf{x}_{i}, \boldsymbol{\xi}_{j}\right\}$ is evaluated with the simulator.

(c) A Kriging model is constructed using the responses from the above step. This step involves solving the MLE problem (12) and selecting the best model by $P R E S S_{R M S}$. 
(d) The accuracy of the Kriging model (Metamodel level 2, figure 1) is assessed based on the predicted $R^{2}$. If the accuracy is not enough, new infill samples $\left(\boldsymbol{\xi}_{\text {infill }}\right)$ are selected in regions with higher predicted error variance. If sufficient accuracy has been achieved then proceed to the following step.

(e) Statistical moments ( $\left.\mathbf{Y}=\boldsymbol{\mu}\left(\mathbf{x}_{i}\right), \boldsymbol{\sigma}\left(\mathbf{x}_{i}\right), \boldsymbol{\gamma}\left(\mathbf{x}_{i}\right), \boldsymbol{\beta}\left(\mathbf{x}_{i}\right)\right)$ are obtained using Monte Carlo simulation assisted by Metamodel level 2, figure 1.

2. Using $\mathbf{X}$ e $\mathbf{Y}$, a Kriging model (Metamodel level 1, figure 1) is fitted for each statistical moment $(\hat{\boldsymbol{\mu}}(\mathbf{x}), \hat{\boldsymbol{\sigma}}(\mathbf{x}), \hat{\gamma}(\mathbf{x}), \hat{\boldsymbol{\beta}}(\mathbf{x}))$.

3. The accuracy of the Kriging model (Metamodel level 1, figure 1) is assessed based on the predicted $R^{2}$. If sufficient accuracy has not been achieved, new infill samples $\left(\mathbf{x}_{\text {infill }}\right)$ are selected in regions with higher predicted error variance. If sufficient accuracy has been achieved then proceed to stage 2 .

Stage 2: Multi-objective optimization

(a) the MORO problem (2) is solved using the evolutionary algorithm (NSGA-II) assisted by the Kriging models obtained in stage 1.

\section{Numerical application}

In this section the proposed methodology is applied to solve the problem of multiobjective robust design of a two-bar truss structure [14]. Different approaches (figure 2) for surrogate-based optimization under uncertainty [8] are used in order to compare their relative merits:

1. Nested approach $(N)$ : the multi-objective optimization is performed on the results of the uncertainty quantification. The statistical moments are obtained using Monte Carlo simulations directly on the simulator. Metamodels are not used at any level. The results obtained by this approach are considered as a reference.

2. Nested/Layered $(N-L)$ : the multi-objective optimization is performed on the results of the uncertainty quantification. The statistical moments are obtained using Monte Carlo simulations on a Metamodel. The metamodel approximates the simulator performance $f(\mathbf{x}, \boldsymbol{\xi})$ in the random variables domain (Metamodel level 2).

3. Layered/Nested/Layered ( $L-N-L)$ (this work) : the multi-objective optimization is performed on a Metamodel (Metamodel level 1 ). The statistical moments are obtained using Monte Carlo simulations on a Metamodel. The metamodel approximates the simulator performance $f(\mathbf{x}, \boldsymbol{\xi})$ in the random variables domain (Metamodel level 2).

Independently of the approach, the multi-objective optimization was carried out using the following parameters of the NSGA-II algorithm: binary tournament selection, number of generations (100), population size (50), crossover probability (0.9), mutation probability (0.1). The Monte Carlo simulations were carried out using 10,000 points. 


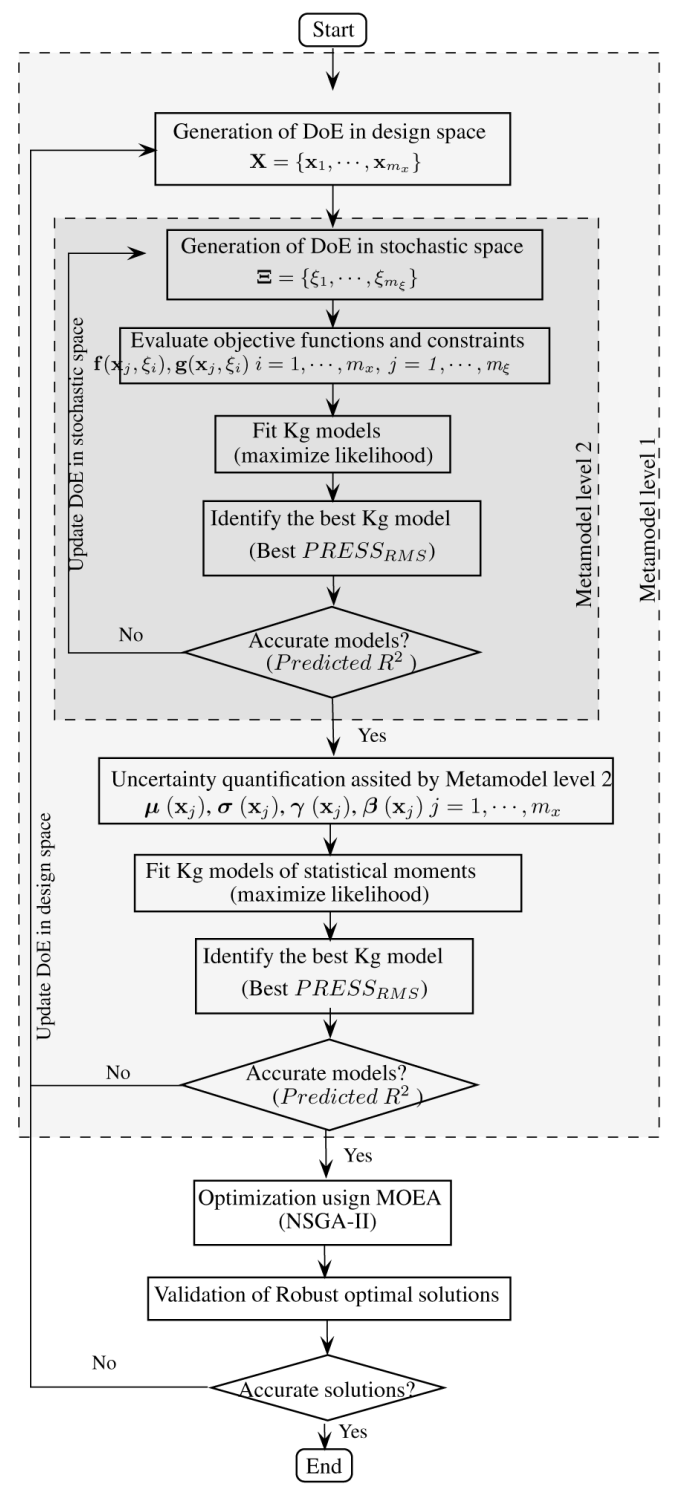

Figure 1: Flowchart of the metamodel-based MORO process.

\subsection{Benchmark problem: two-bar truss structure}

The popular two-bar truss structure problem (figure 3) is used as a benchmark test in this work for the multi-objective robust optimization. The test case is adapted from [14]. The design variables are the cross section diameter $(d)$ and 
the structure height $(H)$. The random parameters of the problem are: the vertical force $(P \sim \mathscr{N}(150,5) \mathrm{kN})$, the structure width $(B \sim \mathscr{N}(750,10) \mathrm{mm})$, the Elastic modulus $\left(E \sim \mathscr{N}(2.1 \mathrm{e} 5,5 \mathrm{e} 3) \mathrm{N} / \mathrm{mm}^{2}\right)$, and the member thickness $(t \sim \mathscr{N}(2.5,0.4) \mathrm{mm})$. The optimization problem is to minimize the volume and the vertical displacement of the structure subject to constraints of stress, buckling as well as the bound constraints (14).

$$
\begin{aligned}
& \min _{d, H}\left\{\mu_{\text {volume }}+k \sigma_{\text {volume }}, \mu_{\text {deflection }}+k \sigma_{\text {deflection }}\right\} \\
& \text { s.t. } g_{1}=\mu_{S}+k \sigma_{S} \leqslant S_{\max } \\
& \quad g_{2}=\mu_{S}+k \sigma_{S} \leqslant S_{\text {crit }} \\
& \quad 20 \leq d \leq 80, \quad 200 \leq H \leq 1,000
\end{aligned}
$$

where:

$$
\begin{aligned}
& S_{\text {max }}=400 M P a, S_{\text {crit }}=\frac{\pi^{2} E\left(t^{2}+d^{2}\right)}{8\left(B^{2}+H^{2}\right)}, S=\frac{P \sqrt{B^{2}+H^{2}}}{2 \pi t d H} \\
& \text { volume }=2 \pi d t \sqrt{B^{2}+H^{2}} \text {, deflection }=\frac{P\left(B^{2} H^{2}\right)^{3 / 2}}{(2 \pi t E d H)^{2}}
\end{aligned}
$$

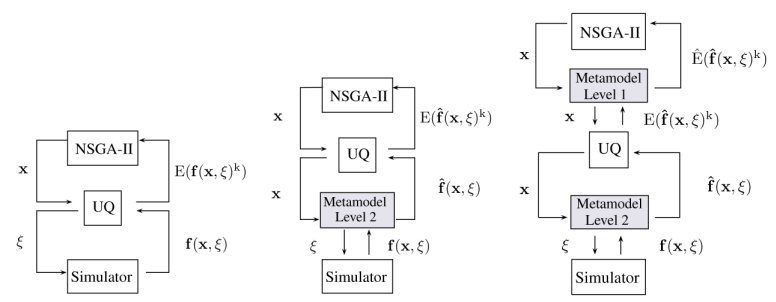

(a)

(b)

(c)

Figure 2: Multiobjective robust optimization approaches. (a) Nested approach, (b) nested/layered, (c) layered/nested/nayered.

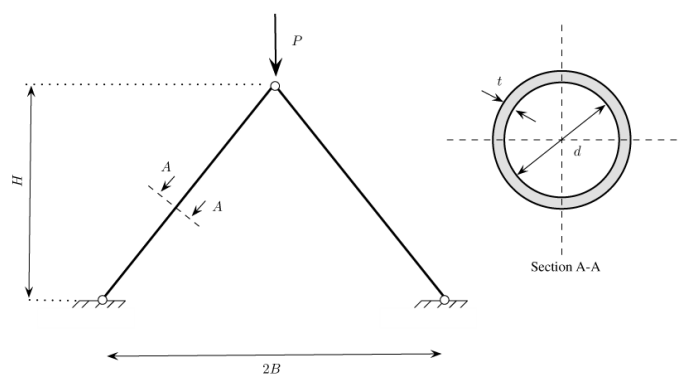

Figure 3: Two-bar truss structure. 


\subsection{Results and discussion}

Twelve Kriging models were constructed. The regression and correlation models are depicted in table 1 . Two LHS of 30 points and 20 points were used to fit the metamodels at levels 1 and 2 respectively. The predicted $R^{2}$ is shown in table 1 along with the $R^{2}$ estimated from Monte Carlo integration at 10,000 points. For this case, the Kriging models have been shown to be highly accurate, with $R^{2}$ values above than 0.98 . Assisted by the former Kriging models, the MORO problem is solved as a deterministic one using the NSGA-II algorithm. The robust Pareto fronts obtained from different approaches and values of $k=1,2$ and 3 are depicted in figure 4 . The Pareto fronts show a good agreement between all the

Table 1: Kriging models.

\begin{tabular}{|l|c|c|c|c|}
\hline & $\begin{array}{c}\text { reg. } \\
\text { order }\end{array}$ & $\begin{array}{c}\text { corr. } \\
\text { func. }\end{array}$ & Pred. $R^{2}$ & $R^{2}$ \\
\hline$f_{1} / \mu_{f_{1}} / \sigma_{f_{1}}$ & $0 / 3 / 3$ & $\mathrm{~g} / \mathrm{e} / \mathrm{eg}$ & $1 / 0.999 / 0.999$ & $1 / 0.999 / 0.999$ \\
\hline$f_{2} / \mu_{f_{2}} / \sigma_{f_{2}}$ & $2 / 3 / 2$ & $\mathrm{~g} / \mathrm{s} / \mathrm{s}$ & $0.999 / 0.969 / 0.986$ & $0.999 / 0.998 / 0.993$ \\
\hline$g_{1} / \mu_{g_{1}} / \sigma_{g_{1}}$ & $3 / 3 / 3$ & $\mathrm{~g} / \mathrm{g} / \mathrm{g}$ & $0.992 / 0.989 / 0.994$ & $0.994 / 0.994 / 0.993$ \\
\hline$g_{2} / \mu_{g_{2}} / \sigma_{g_{2}}$ & $3 / 3 / 3$ & $\mathrm{~g} / \mathrm{g} / \mathrm{g}$ & $0.995 / 0.986 / 0.999$ & $0.999 / 0.999 / 0.991$ \\
\hline
\end{tabular}

$R^{2}$ evaluated by Monte Carlo integration at 10,000 points

correlation: g (gauss), e (exponential), ge (general exponential), s (spline)

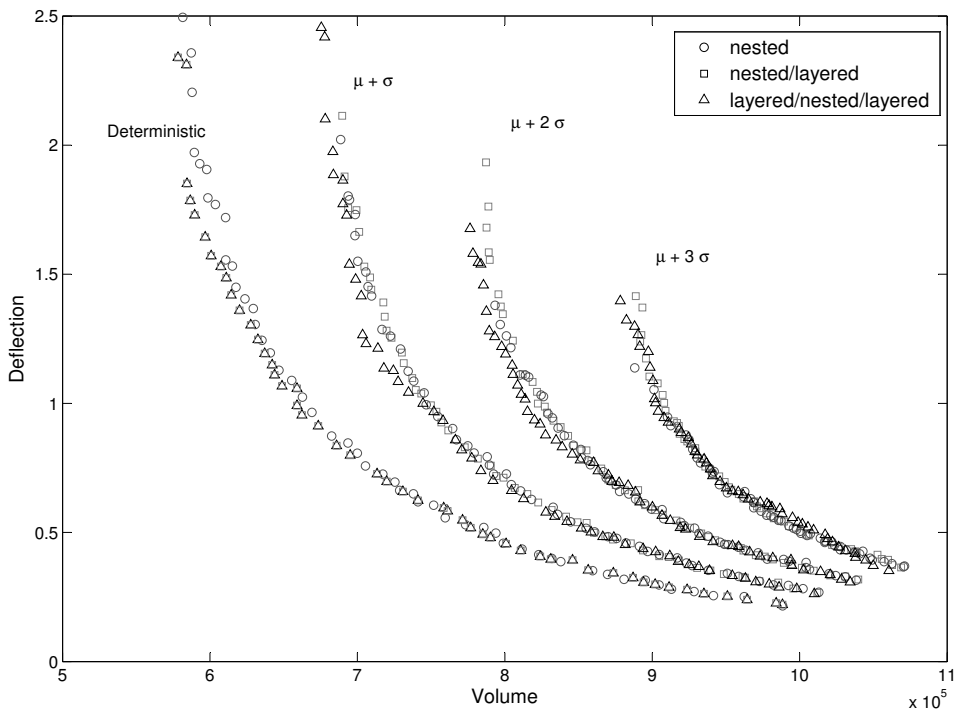

Figure 4: Robust Pareto fronts. 
different approaches. As the volume increases the robust Pareto fronts are closer to the deterministic one, hence the designs are less sensitive to variations in the random parameters. The computational costs of each approach in terms of the number of simulator calls are discussed below:

$$
\begin{aligned}
N_{n} & =N_{\text {pop }} N_{g e n} N_{M C} N_{k}=50 \times 100 \times 10,000 \times 3=1.5 e 8 \\
N_{n / l} & =N_{\text {pop }} N_{g e n} N_{D O E_{M 2}}=50 \times 100 \times 20 \times 3=3.0 e 5 \\
N_{l / n / l} & =N_{D O E_{M 1}} N_{D O E_{M 2}}=30 \times 20=600
\end{aligned}
$$

where $N_{\text {pop }}$ is the population size; $N_{g e n}$ is the number of generations; $N_{M C}$ is the Monte Carlo size; $N_{k}$ is the number of parameter $k$ values; $N_{D O E_{M 1}}$ is the DoE size used in metamodel level 1; and $N_{D O E_{M 2}}$ is the DoE size used in metamodel level 2. The proposed approach allows one to reduce the number of simulator calls by six and three orders of magnitude respectively in comparison to the nested and the nested/layered approaches. It is also worth noting that the proposed approach does not require further simulator runs to obtain Pareto fronts with different levels of robustness.

\section{Conclusion and future prospects}

An approach based on Kriging models to efficiently include the uncertainty quantification in the optimization procedures has been proposed. The proposed methodology allows one: (1) to appraise the robustness of each design using a reduced number of simulator runs compared with conventional methods; (2) to solve the MORO problem in a single-loop optimization assisted by Kriging models; and (3) to obtain multiple solutions to the MORO problem with different levels of robustness of both the objective functions and the constraints, without additional simulator calls.

The results show that the proposed method has the potential to obtain solutions with reasonable accuracy and a considerably lower number of function calls than conventional methods. For the studied case, Kriging models have been shown to be suitable for surrogating the simulator, allowing to estimate the statistical moments efficiently compared with conventional methods. It is also worth noting that the metamodels created during the optimization can be employed in new optimization processes or computationally demanding applications.

The proposed approach is well suited for the multi-objective robust optimization of problems that involve computationally demanding objective functions and/or implicit constraints (FEA). In future works this approach will be applied to higher dimensionality structural problems and different formulations of the MORO problem.

\section{Acknowledgement}

The work presented in this paper was carried out with the support of the Spanish Ministry of Economy and Competitiveness under DPI2011-26394 research project. Its support is greatly appreciated. 


\section{References}

[1] Gunawan, S. and Azarm, S., Multi-objective robust optimization using a sensitivity region concept. Structural and Multidisciplinary Optimization, 29, pp. 50-60, 2005. 10.1007/s00158-004-0450-8.

[2] Deb, K. and Gupta, H., Introducing robustness in multi-objective optimization. Evol Comput, 14, pp. 463-494, 2006.

[3] Li, M., Azarm, S. and Aute, V., A multi-objective genetic algorithm for robust design optimization. Proceedings of the 2005 conference on Genetic and evolutionary computation, ACM: New York, NY, USA, GECCO '05, pp. 771-778, 2005.

[4] Hu, W., Li, M., Azarm, S. and Almansoori, A., Multi-objective robust optimization under interval uncertainty using online approximation and constraint cuts. Journal of Mechanical Design, 133(6), p. 061002, 2011.

[5] Shimoyama, K., Lim, J.N., Jeong, S., Obayashi, S. and Koishi, M., Practical implementation of robust design assisted by response surface approximation and visual data-mining. Journal of Mechanical Design, 131(6), p. 061007, 2009.

[6] Erfani, T. and Utyuzhnikov, S.V., Control of robust design in multiobjective optimization under uncertainties. Structural and Multidisciplinary Optimization, 45, pp. 247-256, 2012.

[7] Mattson, C.A. and Messac, A., Pareto frontier based concept selection under uncertainty, with visualization. Optimization and Engineering, 6, pp. 85-115, 2005.

[8] Eldred, M.S., Giunta, A.A., S. F. Wojtkiewicz, J. and Trucano, T.G., Formulations for Surrogate-Based Optimization Under Uncertainty. Proceedings of the 9th AIAA/ISSMO Symposium on Multidisciplinary Analysis and Optimization, Atlanta, 2002.

[9] Krige, D.G., A statistical approach to some basic mine valuation problems on the witwatersrand. Journal of the Chemical, Metallurgical and Mining Society of South Africa, 52(6), pp. 119-139, 1951.

[10] Kleijnen, J.P., Kriging metamodeling in simulation: A review. European Journal of Operational Research, 192, pp. 707-716, 2009.

[11] Jones, D.R., A taxonomy of global optimization methods based on response surfaces. Journal of Global Optimization, 21, pp. 345-383, 2001.

[12] Sacks, J., Welch, W.J., Mitchell, T.J. and Wynn, H.P., Design and analysis of computer experiments. Statistical Science, 4(4), pp. 409-435, 1989.

[13] Meckesheimer, M., Booker, A.J., Barton, R.R. and Simpson, T.W., Computationally inexpensive metamodel assessment strategies. AIAA Journal, 40, pp. 2053-2060, 2002.

[14] Messac, A. and Ismail-Yahaya, A., Multiobjective robust design using physical programming. Structural and Multidisciplinary Optimization, 23, pp. 357-371, 2002. 10.1007/s00158-002-0196-0. 